STOMACH

\title{
Bax translocation and mitochondrial fragmentation induced by Helicobacter pylori
}

\author{
H Ashktorab, S Frank, A R Khaled, S K Durum, B Kifle, D T Smoot
}

Gut 2004;53:805-813. doi: 10.1136/gut.2003.024372

See end of article for authors' affiliations

Correspondence to:

Dr H Ashktorab, Cancer

Center and

Gastroenterology Division,

Department of Medicine,

Howard University

Hospital, 2041 Georgia

Ave, NW, Washington,

DC 20060, USA;

hashktorab@howard.edu

Accepted for publication

10 December 2003
Background and aims: Previous in vitro and in vivo studies have revealed an association between Helicobacter pylori infection and apoptosis in gastric epithelial cells. Although involvement of the $\mathrm{Bcl}-2$ family of proteins as well as cytochrome $c$ release has been demonstrated in $H$ pylori induced cell death, the exact role of the mitochondria during this type of programmed cell death has not been fully elucidated. Therefore, we sought to determine whether or not Bax translocation and mitochondrial fragmentation occur on exposure of gastric epithelial cells to $H$ pylori, resulting in cell death.

Methods: Experiments were performed with human gastric adenocarcinoma (AGS) cells, AGS cells transfected with the HPV-E6 gene (which inactivates p53 function), AGS-neo cells (transfected with the backbone construct), mouse embryonic fibroblasts (MEFs), and p19 ${ }^{A R F}$ null $\left(\mathrm{ARF}^{-/}{ }^{-}\right.$) MEFs. Cells were incubated with a cag positive $H$ pylori strain for up to 24 hours, lysed, and cytoplasmic and mitochondrial membrane fractions were analysed by western blot for Bax translocation.

Results: Bax translocation was detected in AGS, AGS-neo, and normal MEF cells after exposure to $H$ pylori for three hours, but not in $\mathrm{ARF}^{-/-} \mathrm{MEFs}$ cells. Translocation of Bax after $\mathrm{H}$ pylori incubation was also detected in AGS-E6 cells (inactive p53 gene) but to a lesser degree than in AGS-neo cells. In parallel studies, the mitochondrial morphology of living cells infected with $H$ pylori was assessed by confocal microscopy. Mitochondrial fragmentation was detectable after 10 hours of $H$ pylori incubation with AGS cells and after seven hours with MEF cells. In wild-type MEFs, mitochondrial fragmentation was significantly increased in comparison with ARF null MEFs (43\% v 10.4\%, respectively). Furthermore, mitochondrial depolarisation and caspase-3 activity were initiated within four hours in cells incubated with $H$ pylori, and these events were inhibited by forced expression of $\mathrm{Bcl}-2$.

Conclusions: These data suggest that during $H$ pylori induced apoptosis, Bax translocates to the mitochondria which subsequently undergo depolarisation and profound fragmentation. Functional ARF and p53 proteins may play an important role in $\mathrm{H}$ pylori induced mitochondrial modification.
A pproximately 24000 new cases of gastric cancer are diagnosed each year in the USA and there are about 700000 new cases diagnosed worldwide. There will be an estimated 12400 deaths from this type of cancer in 2003 in the USA. Several studies have provided evidence that Helicobacter pylori is a major risk factor for gastric carcinogenesis. $H$ pylori has been shown to induce apoptosis in gastric epithelial cells in vitro and in vivo. ${ }^{1-6}$ In vitro studies have demonstrated that $H$ pylori can induce apoptosis in different gastric epithelial cell lines. ${ }^{2-10}$ These studies have demonstrated that several $H$ pylori factors, including products of the cag pathogenicity island, ${ }^{11}$ urease, $^{12}$ and lipopolysaccharide ${ }^{13}$ contribute to apoptosis. The extent of apoptosis induced by $H$ pylori may be dependent on several factors, including cell type, $H$ pylori strain, and culture conditions.

The apoptotic process often involves the mitochondria. When involved, the mitochondrial outer membrane is permeabilised and/or ruptured. ${ }^{14}{ }^{15}$ As a result, proapoptotic proteins normally confined to the intermembrane space are released into the cytosol. ${ }^{16}$ Protein translocation from mitochondrial localisation triggers catabolic reactions which results in cell death. For example, cytochrome $c$ release from the mitochondrion to the cytosol activates the caspase-3/9 complex. ${ }^{17}$ Caspases are aspartate specific cysteine proteases that play an important role in apoptosis. ${ }^{1819}$ Activation of downstream caspases are preceded by loss of mitochondrial membrane potential and release of cytochrome $c^{20}$ In addition, cytochrome $c$ and other proteins are released from the mitochondria into the cytosol. ${ }^{21} 22$
Apoptosis is regulated by proteins from the Bcl-2 family encoding proteins that can either inhibit (for example, Bcl-2, $\mathrm{Bcl}-\mathrm{X}_{\mathrm{L}}$ ) or promote (for example, Bax, Bcl- $\mathrm{X}_{\mathrm{S}}$, Bak) apoptosis. ${ }^{23}$ The ratio at which these proteins are expressed can determine whether or not a cell undergoes apoptosis. Permeabilisation of mitochondrial membranes, a decisive feature of early cell death, is regulated by members of the Bcl2 family which interact with the permeability transition pore complex. The cytoprotective oncoprotein Bcl-2 stabilises mitochondrial membrane barrier function whereas the tumour suppressor protein Bax permeabilises mitochondrial membranes. Regulation of membrane permeabilisation is intertwined with that of the bioenergetic and redox functions of mitochondria. ${ }^{24}$ Truncated BID (tBID) functions as a membrane targeted and concentrated death protein. Once tBID is activated, it induces oligomerisation of BAK. Both Bid and Bak knockout mice studies have shown the importance of tBID in the release of cytochrome $c .^{23}$ Hence tBID induces its intramembranous oligomerisation into a proposed pore for cytochrome $c$ efflux. The BH3 domain of tBID remains on the mitochondrial surface where it is required to trigger BAK to release cytochrome $c$. The proapoptotic member BAX, which is highly homologous to BAK, rapidly forms pores in

Abbreviations: AGS, human gastric adenocarcinoma; MEFs, mouse embryonic fibroblasts; GFP, green fluorescent protein; HPV, human papilloma virus; PARP, poly adenosine ribose polymerase; TMRE, tetramethylrhodamine; DMEM, Dulbecco's modified Eagle's medium; PBS, phosphate buffered saline; SDS-PAGE, sodium dodecyl sulphatepolyacrylamide gel electrophoresis 
liposomes that release cytochrome $c .^{25}$ Thus an activation cascade of proapoptotic proteins from BID to BAK or BAX integrates the cell death pathway leading to the irreversible efflux of cytochrome $c^{23}$ Bax, Bcl-2, and Bcl- $\mathrm{X}_{\mathrm{L}}$ contain a carboxy terminal hydrophobic domain that has been presumed to function as a membrane anchor domain. ${ }^{26}$ Experiments have indicated that Bax translocates from the cytoplasm to the mitochondrial membrane on activation of apoptosis and that Bax is a good candidate for mediating release of mitochondrial proteins. ${ }^{26}$ Therefore, we set up the following experiments to study the role of Bax in altering mitochondrial dynamics in gastric epithelial cells undergoing apoptosis after exposure to $H$ pylori.

$H$ pylori induced apoptosis is associated with an increase in Bak expression in both gastric biopsies from patients colonised with $H$ pylori and in vitro. ${ }^{2}$ Konturek et al showed induction of apoptosis with evidence of Bax upregulation and Bcl-2 downregulation in $H$ pylori positive duodenal ulcer patients. ${ }^{7}$ The $\mathrm{Bcl}-2$ family, particularly $\mathrm{Bcl}-\mathrm{X}_{\mathrm{L}}$, are major antiapoptotic factors. Bax is a proapoptotic factor which may cross talk with p53. ${ }^{27}$ Bax redistributes from the cytoplasm to the mitochondria during cell death. ${ }^{26}{ }^{29}$ Moreover, studies have shown that $H$ pylori mediated apoptosis may occur through several pathways involving activation of $\mathrm{p} 53,{ }^{8}$ the death receptor Fas, and the death ligand tumour necrosis factor $\alpha^{29}{ }^{30}$

Levels of p53 protein are tightly controlled within eukaryotic cells. The rise in p53 protein levels, in response to cellular stress or DNA damage leading to apoptosis, is frequently a post-translational event resulting from decreased degradation. Break down of p53 can occur through its binding to MDM2, which targets p53 for ubiquitin proteosomal degradation. ${ }^{31}{ }^{32} \mathrm{P}_{1} 9^{\mathrm{ARF}}$ (pl $4^{\mathrm{ARF}}$ in humans) is upstream of p53 and when bound to p53 inhibits MDM2 mediated degradation of p53. ${ }^{32} 33$ ARF can also bind p53 and enhance its transcriptional activity. Thus activation of $\mathrm{p} 14^{\mathrm{ARF}}$ can promote p53 mediated apoptosis within eukaryotic cells. We have recently shown that $\mathrm{p} 53$ sensitises cells to apoptosis induced by $\mathrm{H}$ pylori. ${ }^{34}$ Also, our experiments showed involvement of ARF in stabilising p53 in cells exposed to $\mathrm{H} \mathrm{pylori.}^{34}$

The relationship between $H$ pylori induced apoptosis with ulcer and cancer is not very clear. It is possible that cancer may result from Bcl-2 overexpression in the presence of an inactive Bax (possibly through mutation). Pathways that $H$ pylori infection uses for inducing apoptotic host cell mechanisms are still unknown. Mitochondria disintegration and dynamic changes in apoptotic cells have been reported. ${ }^{35}$ Fragmentation of mitochondria has been observed on Bax overexpression during apoptosis. ${ }^{36}$ The objectives of this study were to determine if there are dynamic changes in mitochondria in gastric cells exposed to $H$ pylori, and to elucidate whether or not p53 is involved in mitochondrial modification induced by $H$ pylori. These experiments demonstrated that on $H$ pylori incubation, Bax translocates to the mitochondria followed by membrane depolarisation, and mitochondrial fragmentation. These phenomena are reduced in cells lacking functional wild-type p53. The reduction in Bax translocation in cells lacking p53 varies between cell types. P53 cross talk with Bax in these cells may influence cell death through mitochondrial fragmentation but it is not critical as gastric cells that lack wild-type p53 still undergo apoptosis.

\section{MATERIALS AND METHODS \\ Cell culture}

A gastric adenocarcinoma cell line (AGS) purchased from the American Type Culture Collection (Manassas, Virginia, USA) was used for these experiments. AGS cells were cultured in Dulbecco's modified Eagle's medium (DMEM) supplemented with $10 \%$ fetal bovine serum without antibiotics. Cells were incubated in $5 \% \mathrm{CO}_{2}$ at $37^{\circ} \mathrm{C}$ in humidified air. Mouse embryonic fibroblasts (MEF) were cultured in DMEM supplemented with $10 \%$ fetal bovine serum (a gift from Dr Karen Vousden, Frederick, Maryland, USA).

\section{Infection and transfection of cell lines}

Retroviral infection of AGS cells

AGS cells were grown to 50\% confluency in a $100 \mathrm{~mm}$ culture dish. Medium was removed and replaced with $3 \mathrm{ml}$ of $4 \mu \mathrm{g} / \mathrm{ml}$ polybyrene and $1 \mathrm{ml}$ of virus stock (human papillomavirus (HPV) type 16 containing the E6 gene or an empty vector, Neo). After incubating AGS cells with virus for two hours at $37^{\circ} \mathrm{C}$, an additional $5 \mathrm{ml}$ of culture medium with polybyrene were added to the culture dish for another five hours of incubation. Cells were then rinsed with phosphate buffered saline (PBS) and $10 \mathrm{ml}$ of medium without polybyrene were added. On the second day, cells were split $1 / 10$ to $1 / 200$ in order to isolate individual colonies. On the third day, medium was replaced with medium containing G4l8 at a concentration of $400 \mu \mathrm{g} / \mathrm{ml}$ for selection. Stable colonies (AGS-E6 and AGS-neo) were isolated and used in these experiments.

Transient transfections of AGS, AGS-E6, and AGS-neo with Bcl-2 (pcDNA3-Bcl-2 was a gift from Dr Carlo Croce, Thomas Jefferson University) were performed using Fugene 6 (Roche, Indianapolis, Indiana, USA) following the manufacturer's protocol. Based on green fluorescent protein (GFP) expression, the transfection efficiency was close to $80 \%$. Cells were incubated overnight, washed, and infected with $H$ pylori, as described below.

\section{Confocal microscopy}

AGS and MEF cells, respectively, were grown in $4.3 \mathrm{~cm}^{2}$ chamber slides (two well Lab-Tek chambered coverglass system; Nalge Nunc Inc., Naperville, Illinois, USA) and transfected with Lipofectamine 2000 transfection reagent (Invitrogen, Carlsbad, California, USA) according to the manufacturer's instructions using $2.0 \mu \mathrm{g}$ of mito-GFP (Clontech Laboratories, Inc., Palo Alto, California, USA) per chamber.

\section{H pylori culture}

Cag positive $H$ pylori strain J117 (a gift from Dr Timothy Cover $^{37}$ ) was cultured on trypticase soy agar with 5\% sheep's blood (Curtin Matheson, Jessup, Maryland, USA) with Skirrow's selective antibiotic supplement (Prolab Inc., Scarborough, Canada) at $37^{\circ} \mathrm{C}$ in a $\mathrm{CO}_{2} / \mathrm{O}_{2}$ water jacketed incubator (Forma Scientific, Marietta, Ohio, USA) under microaerophilic conditions $\left(10 \% \mathrm{CO}_{2}, 7.5 \% \mathrm{O}_{2}, 82.5 \% \mathrm{~N}_{2}\right) . H$ pylori were added to cells at a bacteria:cell concentration of 100:1, based on previous reports that $H$ pylori reproducibly induces apoptosis in AGS cells at this ratio. ${ }^{96}$ Cultures were maintained for three days prior to passing; bacteria were used between passages 5 and 15 for these experiments to ensure that the bacteria were able to readily adhere to AGS cells. Adherence was visualised using microscopy.

\section{Mitochondrial isolation and Bax translocation analysis}

AGS, AGS-E6, or AGS-neo cells were incubated with $H$ pylori for three and six hours and then assayed for Bax translocation to the mitochondria. Lysis of cells for mitochondrial protein extraction was performed in isotonic buffer $(200 \mathrm{mM}$ mannitol, $70 \mathrm{mM}$ sucrose, $1 \mathrm{mM}$ EGTA, $10 \mathrm{mM}$ HEPES, pH 6.9) by dounce homogenisation. Unbroken cells, nuclei, and heavy membranes were pelleted at $1000 \mathrm{~g}$ for five minutes and discarded. The mitochondrial enriched fraction was then produced by pelleting at $12000 \mathrm{~g}$ for 20 minutes. 
The pellet was then washed briefly in alkaline wash buffer (0.1 $\mathrm{M} \mathrm{Na}_{2} \mathrm{CO}_{3}, \mathrm{pH}$ 11.5) to separate peripherally associated (alkali sensitive) mitochondrial proteins from membrane integrated (alkali resistant) mitochondrial proteins and centrifuged. The pellet (containing the membrane integrated proteins) was then resuspensed in RIPA lysis buffer $(1 \times$ PBS, $1 \%$ Nonidet P-40, $0.5 \%$ sodium deoxycholate, $0.1 \%$ sodium dodecyl sulphate) with protease inhibitor cocktail (Calbiochem, San Diego, California, USA) and used for immunoblotting.

For detection of Bax and cytochrome $c$ oxidase protein (cytochrome $c$ oxidase, subunit IV, detection was employed as a control to demonstrate that mitochondrial protein fractionation was successfully achieved) by western blot, equivalent samples $(20 \mu \mathrm{l}$ containing approximately $20 \mu \mathrm{g}$ protein) were separated by sodium dodecyl sulphate-polyacrylamide gel electrophoresis (SDS-PAGE) on 12\% Tris glycine gels (Invitrogen) and transferred to $0.2 \mathrm{mM}$ polyvinylidene difluoride membranes (Invitrogen). Blots were probed with a rabbit polyclonal antiserum specific for the amino terminal of Bax (N20; Santa Cruz, California, USA) or with a mouse polyclonal antibody specific for cytochrome $c$ oxidase (Research Diagnostics, Flanders, New Jersey, USA), followed by the appropriate secondary antibodies conjugated to horseradish peroxidase (Santa Cruz) and then visualised by ECL kit (Pierce, Rockford, Illinois, USA) following the manufacturer's protocol.

\section{Measurement of mitochondrial membrane potential, viability, and caspase activity}

To measure mitochondrial membrane potential $\left(\Delta \psi_{m}\right)$, AGS, AGS-E6, or AGS-neo cells $\left(1 \times 10^{6}\right.$ cells $\left./ \mathrm{ml}\right)$ were resuspended in a $10 \mu \mathrm{g} / \mathrm{ml}$ solution of the cationic lipophilic dye tetramethylrhodamine ethyl ester (TMRE) (Molecular Probes, Eugene, Oregon, USA). For estimation of $\Delta \psi_{m}$, cells were incubated with $100 \mathrm{nM}$ TMRE for 15 minutes at room temperature in PBS. After incubation, cells were immediately analysed by flow cytometry. Dead cells were excluded by forward and side scatter gating. Data were accumulated by analysing an average population of 20000 cells. TMRE fluorescence was detectable in the PI channel (red fluorescence, emission at $590 \mathrm{~nm}$ ). Caspase-3 activity was measured using PhiPhiLuxG1D2 kit (Calbiochem), a peptide substrate for caspase-3, which when cleaved emits green fluorescence (excitation, $505 \mathrm{~nm}$; emission, $530 \mathrm{~nm}$ ). Results were assayed by flow cytometry. Propidium iodide (PI) staining of non-viable cells was performed by incubation with $1 \mu \mathrm{l} / 10^{6}$ cells of PI solution ( $2 \mathrm{mg} / \mathrm{ml}$ in PBS) for $>5$ minutes. Stained

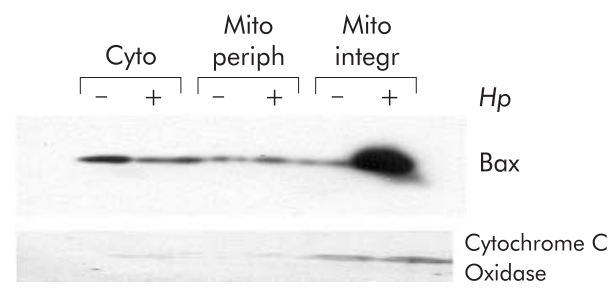

Figure 1 Helicobacter pylori induced mitochondrial translocation of Bax. AGS cells were cocultured with $\mathrm{H}$ pylori for three hours $(+)$ or cultured in medium alone as a control $(-)$. Cytosolic (Cyto), mitochondrial peripheral (Mito periph), and integral (Mito integr) proteins were loaded onto sodium dodecyl sulphate-polyacrylamide gels followed by immunoblotting for Bax and cytochrome $c$ oxidase. Mitochondrial translocation of Bax is shown in samples treated with $H$ pylori. There was a decrease in cytosolic Bax in AGS cells incubated with $\mathrm{H}$ pylori and a concomitant increase in mitochondrial membrane integrated Bax. The presence of the control protein, cytochrome $c$ oxidase, in the mitochondrial membrane fractions confirms the quality of the lysates. cells were analysed by flow cytometry with excitation at $488 \mathrm{~nm}$ and emissions at $>550 \mathrm{~nm}$.

\section{Confocal microscopy and mitochondria fragmentation}

Cells (gastric and MEF cells) were overlaid with culture medium with or without $H$ pylori and incubated for up to 10 hours. Two well chamber slides which allow parallel and comparative imaging of two separately transfected cell populations under identical experimental conditions were used throughout the study. To prevent cell detachment and to better maintain cellular morphology, cells were pretreated with the caspase inhibitor Z-VAD-fmk $(25 \mu \mathrm{M}$; Alexis Biochemicals, San Diego, California, USA) and $10 \mathrm{mM}$ HEPES buffer (pH 7.0). Quantitative experiments have shown that there is no difference in the mitochondrial fragmentation rate of Z-VAD-fmk pretreated cells compared with controls. Images prior to and at given time points after $H$ pylori exposure were collected under thermostated conditions $\left(37^{\circ} \mathrm{C}\right)$ using an Olympus Fluoview confocal microscope (FV300-IX70) with an $60 \times$ UPlan apochromatic objective (Olympus, Melville, New York, USA). The $488 \mathrm{~nm}$ line of an argon laser was used for fluorescence excitation of enhanced GFP. After acquisition, images were processed using Fluoview and Photoshop software.

All experiments aimed at assessment and quantification of mitochondrial phenotypes were repeated at least twice independently. Typically, approximately 150 randomly selected cells were assessed per single experiment. A punctiform mitochondrial phenotype after $H$ pylori incubation was scored when at least $90 \%$ of the tubular mitochondria were disintegrated.

\section{Statistics}

For analysis of paired observations before and after $H$ pylori exposure, we used a paired $t$ test for normally distributed data. Each experiment was repeated three times and $\mathrm{p}<0.05$ was considered significant.

\section{RESULTS}

\section{$H$ pylori mediated Bax translocation}

$H$ pylori is a known inducer of cell death but the particulars of this death pathway are not fully understood. To begin to dissect how $H$ pylori triggers cell death, we analysed mitochondrial translocation of the death protein Bax. Mitochondrial translocation of Bax can be observed within three hours of incubation of $H$ pylori with AGS cells (fig 1). Next, we asked if mitochondrial translocation of Bax was dependent on ARF and p53. AGS-E6 (lacking functional p53), AGS-neo (control), and AGS (wild-type p53) cells were incubated with $H$ pylori for three hours. HPV-E6 has been shown to efficiently target p53 for degradation through MDM2 independent mechanisms, and E6 expressing cells are unable to stabilise p53 in response to cell stress or DNA damage making them functionally p53 null. ${ }^{38}{ }^{39}$ AGS-neo cells were used as a negative control as they carry the backbone vector without E6 which should have no effect on p53 function. Bax translocated to the mitochondria in both AGS and AGS-neo control cells (fig 2). Partial translocation of Bax to the mitochondria was observed in AGS-E6 cells which are p53 null (fig 2). We scanned and calculated the degree of Bax translocation in all of the cells treated and untreated with $H$ pylori. The level of Bax translocation in AGS, AGS-neo, and AGS-E6 treated cells were 2.4-, 2.2-, and 1.3fold $(p<0.05)$, respectively. Note that basal levels of mitochondrial Bax in uninfected AGS cells are higher in fig 2 compared with fig 1 due to differences in loading between experiments. However, this still does not account for all of the increase in mitochondrial Bax content in infected cells. These results suggest that $H$ pylori induces activation of 


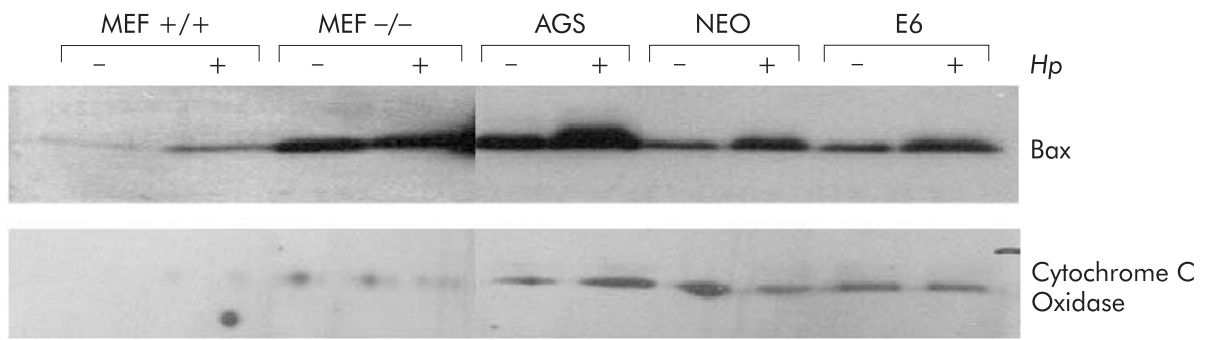

Figure 2 Helicobacter pylori induced mitochondrial Bax translocation is dependent on ARF and partially on p53. Mouse embryonic fibroblasts (MEF) from either $\mathrm{ARF}^{+/+}$or $\mathrm{ARF}^{-/-}$mice, AGS, AGS-E6 (E6), and AGS-neo (NEO) cells were cocultured with $\mathrm{H}$ pylori for three hours (+) or cultured in medium alone as a control (-). Cytosolic and mitochondrial extracts were loaded onto sodium dodecyl sulphate-polyacrylamide gels followed by immunoblotting for Bax and cytochrome $c$ oxidase. Bax translocated was integrated into mitochondrial membranes in ARF ${ }^{+/+}$MEFs, AGS, and AGS-neo (3-, 2.4-, and 2.2-fold, respectively) but not into ARF ${ }^{-1-}$ MEFs, and to a minimum in AGS-E6 cells (1.3-fold), suggesting that ARF, and to some extent p53, contribute to activation of Bax. Cytochrome $c$ oxidase used as an internal control confirms the loading and quality of the lysates.

Bax, targeting the mitochondrial membrane, and that this process involves p53 dependent and independent pathways.

In prior studies we have shown that mouse embryonic fibroblasts (MEFs) are as sensitive as AGS cells to $H$ pylori induced apoptosis. Also, $\mathrm{ARF}^{-1-}$ and $\mathrm{p} 53^{-1-}$ MEFs are more resistant to $H$ pylori induced apoptosis than wild-type MEFs. ${ }^{34}$ In additional experiments MEFs from wild-type and $\mathrm{ARF}^{-/-}$ mice were incubated with $H$ pylori for three hours. Bax translocated and integrated into mitochondrial membranes in $\mathrm{ARF}^{+/+}$MEFs but not in $\mathrm{ARF}^{-/-}$MEFs, suggesting that ARF contributes to activation of Bax, possibly through stabilisation of p53 (fig 2). Basal levels of Bax in $\mathrm{ARF}^{-1-}$ cells appear artificially high. This basal "mitochondrial" BAX could be peripherally attached to mitochondria in a large oligomer, while "active" BAX which damages mitochondria is integrated into the membrane. In fact, the mitochondrial lysates were only enriched for mitochondria and could also contain other light membranes (for example, endoplasmic reticulum).

\section{Mitochondrial depolarisation is caused by $\mathrm{H}$ pylori infection and is blocked by $\mathrm{Bcl}-2$}

Incubation of cells with $H$ pylori provokes profound damage to mitochondria. To further assess the death pathway targeting mitochondria, we measured mitochondrial membrane depolarisation as a marker of mitochondrial dysfunction, using the fluorescent dye TMRE. As shown in fig 3, mitochondria begin to depolarise within 4-5 hours of incubation with $H$ pylori, as measured by loss of fluorescence. Mitochondrial membrane potential decreases in p53 containing cell lines, AGS and AGS-neo, as well as in p53 null AGSE6 cells. Hence there are also p53 independent death effects
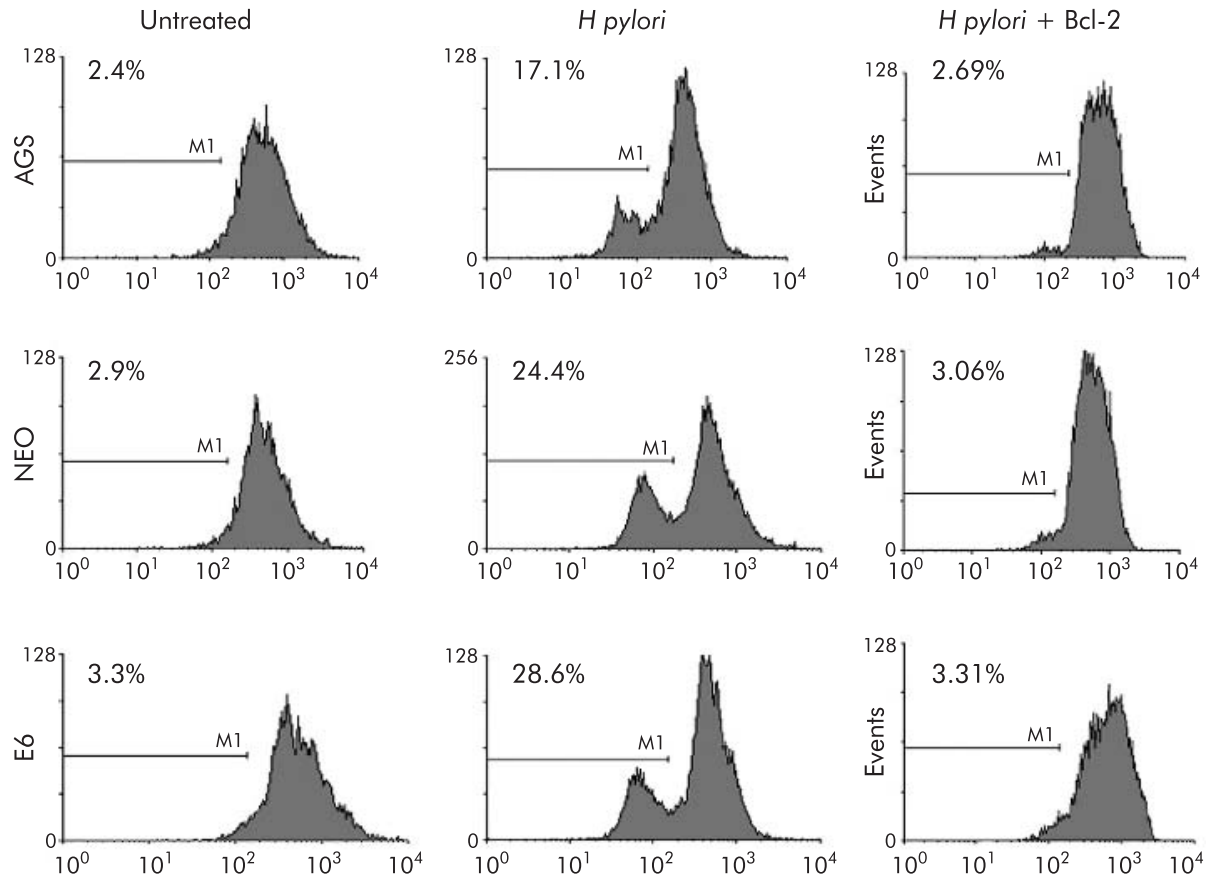

Figure 3 Loss of mitochondrial membrane potential $\left(\Delta \psi_{\mathrm{m}}\right)$ occurred in gastric cells treated with Helicobacter pylori, and mitochondrial membrane potential loss was inhibited by forced Bcl-2 expression. AGS, AGS-E6 (E6), or AGS-neo (NEO) cells $\left(1 \times 10^{6} \mathrm{cell} / \mathrm{s} / \mathrm{ml}\right.$ ) were resuspended in $10 \mu \mathrm{g} / \mathrm{ml}$ tetramethylrhodamine (TMRE). After incubation, cells were immediately analysed by flow cyłometry. Dead cells were excluded by forward and side scatter gating. Data were accumulated by analysing an average population of 20000 cells. TMRE fluorescence was detectable in the PI channel (red fluorescence, emission at $590 \mathrm{~nm}$ ). In parallel experiments AGS, AGS-E6, or AGS-neo cells transfected with Bcl-2 (to inhibit apoptosis) were stained as explained above. Loss of $\Delta \psi_{m}$ was quantified by flow cytometry analysis of untransfected cells versus $\mathrm{Bcl}-2$ transfected gastric cells after $H$ pylori treatment for the indicated time periods. Bcl-2 transfected cells exhibited a significantly reduced $\Delta \psi_{m}$ at four hours after $H$ pylori treatment ( $\left.p<0.05\right)$. In contrast, Bcl-2 transfected cells exhibited a much slower decline in membrane potential. 

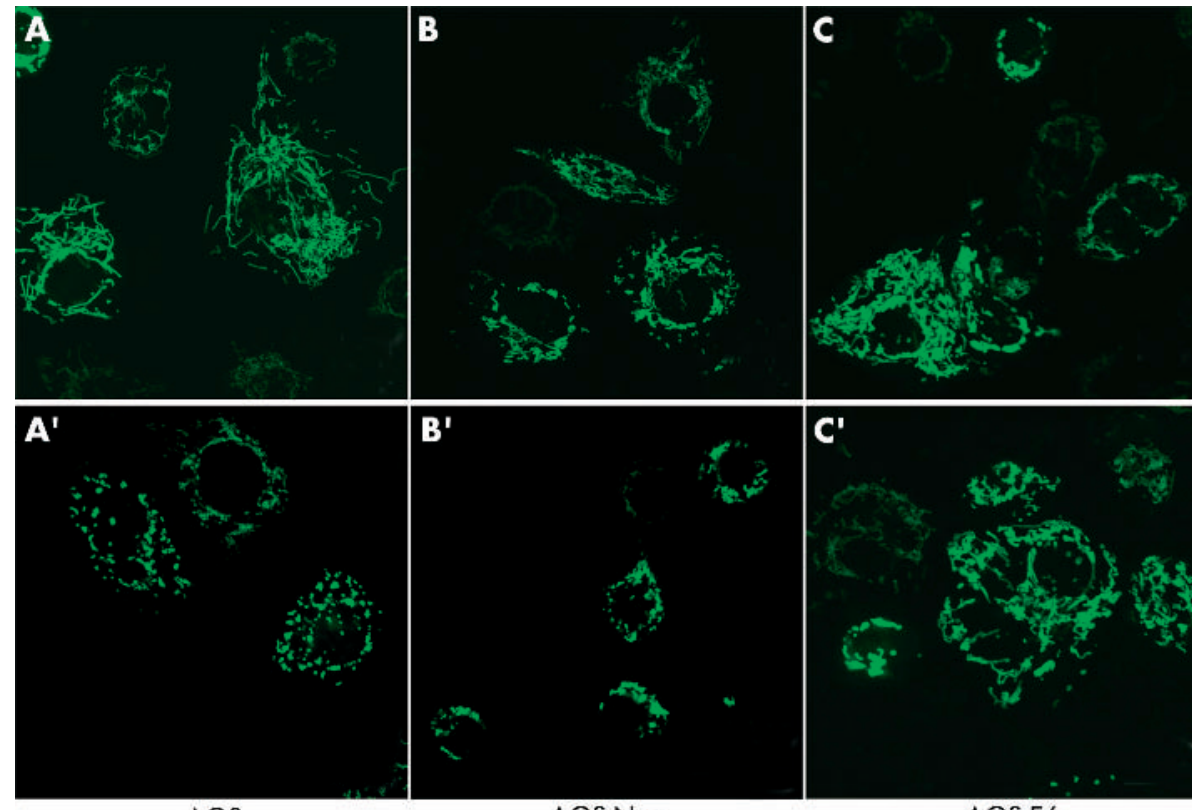

AGS

AGS-Neo

AGS-E6

Figure 4 AGS, AGS-E6, and AGS-neo cells transfected with mito-green fluorescent protein (GFP) were treated with Helicobacter pylori and visualised by confocal microscopy. Mitochondrial morphology was assessed after $\mathrm{H}$ pylori treatment. At 10 hours of treatment, more than $70 \%$ (5\%) of AGS cells showed a fragmented mitochondrial phenotype $\left(\mathrm{A}^{\prime}\right)$. Comparable results were obtained in AGS-neo cells $\left(\mathrm{B}^{\prime}\right)$. The typical reticulotubular mitochondrial phenotype of healthy AGS cells disintegrated into multiple small rounded organelles $\left(A, A^{\prime}\right)$. In contrast, in AGS-E6 cells with inactivated p53 function, less than $15 \%(5 \%)$ of cells underwent mitochondrial fragmentation under identical experimental conditions $\left(C, C^{\prime}\right)$.
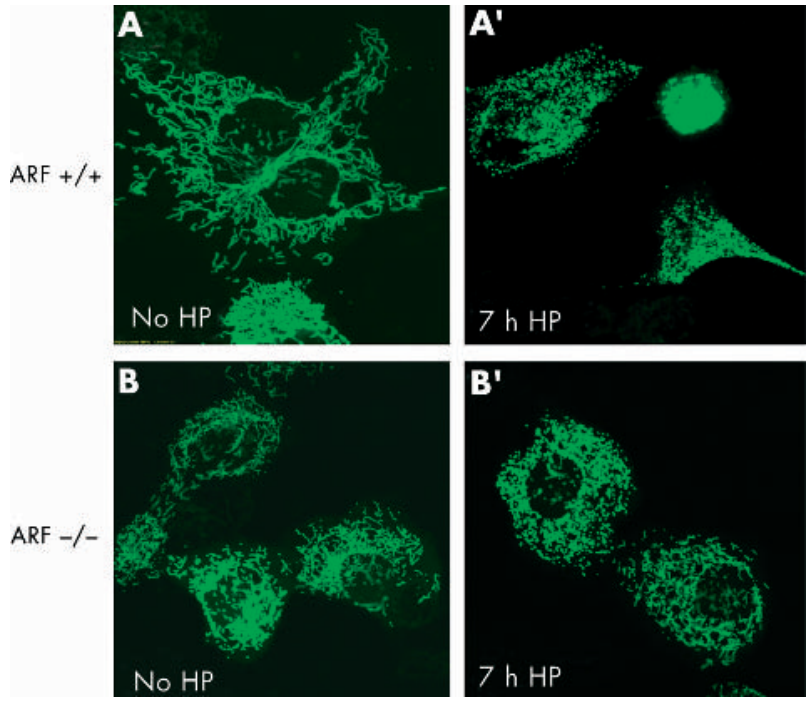

Figure 5 Helicobacter pylori induced mitochondrial fragmentation. $\mathrm{ARF}^{+/+}$mouse embryonic fibroblasts (MEFs) and $\mathrm{ARF}^{-/-} \mathrm{MEFs}$ transfected with mito-green fluorescent protein (GFP) were treated with $H$ pylori and visualised by confocal microscopy. Mitochondrial morphology of $\mathrm{ARF}^{+/+} \mathrm{MEFs}$ cells was assessed at seven hours after $H$ pylori treatment by confocal microscopy. The gradual decrease in the percentage of cells featuring normal mitochondrial morphology was paralleled by an increasing percentage of cells exhibiting the punctiform phenotype. $\left(\mathrm{A}^{\prime}\right) \mathrm{ARF}^{+/+} \mathrm{MEFs}$ undergoing profound changes in their mitochondrial phenotype during $\mathrm{H}$ pylori induced apoptosis are shown. In $\mathrm{ARF}^{+/+} \mathrm{MEF}$ cells, the characteristic reticulotubular mitochondrial morphology $(A)$ disintegrates into numerous round fragments of varying size $\left(A^{\prime}\right)$. In some cells, as exemplified by the bottom panel, the resulting punctiform mitochondria appear swollen, relative to the normally thin diameter of the mitochondrial tubules. (B) Mitochondrial morphology was also documented in mito-GFP transfected $\mathrm{ARF}^{-/-} \mathrm{MEFs}$ cells before (B) and after seven hours of $H$ pylori treatment $\left(B^{\prime}\right)$. Mitochondria appear healthy and no obvious morphological changes were observed. in these cells, induced by $H$ pylori, targeting the mitochondria. To determine if mitochondrial damage mediated by $H$ pylori is mediated by Bax, we transiently expressed the antiapoptotic protein $\mathrm{Bcl}-2$ prior to incubation with $H$ pylori. The balance between Bax and Bcl-2 is known to regulate apoptosis. ${ }^{27}{ }^{40}$ As others have shown that Bcl-2 does not block Bax translocation $^{17}$ but protects mitochondria from Bax mediated damage, ${ }^{41}{ }^{42}$ we measured mitochondrial depolarisation in cells incubated with $H$ pylori. Forced expression of Bcl-2 was able to protect the mitochondria of cells incubated with $H$ pylori from depolarisation. Thus the apoptotic pathways induced by $H$ pylori in AGS cells activate Bax, disrupt mitochondrial integrity, and are abrogated by Bcl-2.

\section{Fragmentation of mitochondria by $\mathrm{H}$ pylori}

To test whether or not apoptotic cell death caused by $H$ pylori was associated with changes in the mitochondrial phenotype, AGS, AGS-E6, and AGS-neo cells were transiently transfected with a green fluorescent protein/cytochrome $c$ oxidase subunit VIII fusion construct (mito-GFP) that specifically targets the mitochondrial matrix compartment. ${ }^{43}$ At 12 hours after transfection, cells were incubated with $H$ pylori at 100 bacteria per cell and mitochondrial morphology of living cells was subsequently assessed by confocal microscopy. Although confocal imaging of the mitochondrial network of AGS is hampered by the fact that these comparatively small cells round up very early during cell death, profound changes in mitochondrial morphology were readily seen after $H$ pylori incubation. As exemplified by images $\mathrm{A}$ and $\mathrm{A}^{\prime}$ in fig 4, the typical reticulotubular mitochondrial phenotype of healthy AGS cells disintegrated into multiple small rounded organelles. At 10 hours after infection, more than $70 \%(5 \%)$ of AGS cells showed a fragmented mitochondrial phenotype. Comparable results were obtained in AGS-neo cells (fig 4, images $\left.\mathrm{B}, \mathrm{B}^{\prime}\right)$. In contrast, in AGS-E6 cells with inactivated p53 function, less than $15 \%$ (5\%) of cells underwent mitochondrial fragmentation under identical experimental conditions (fig 4, images $C, C^{\prime}$ ). In addition, we noted that 

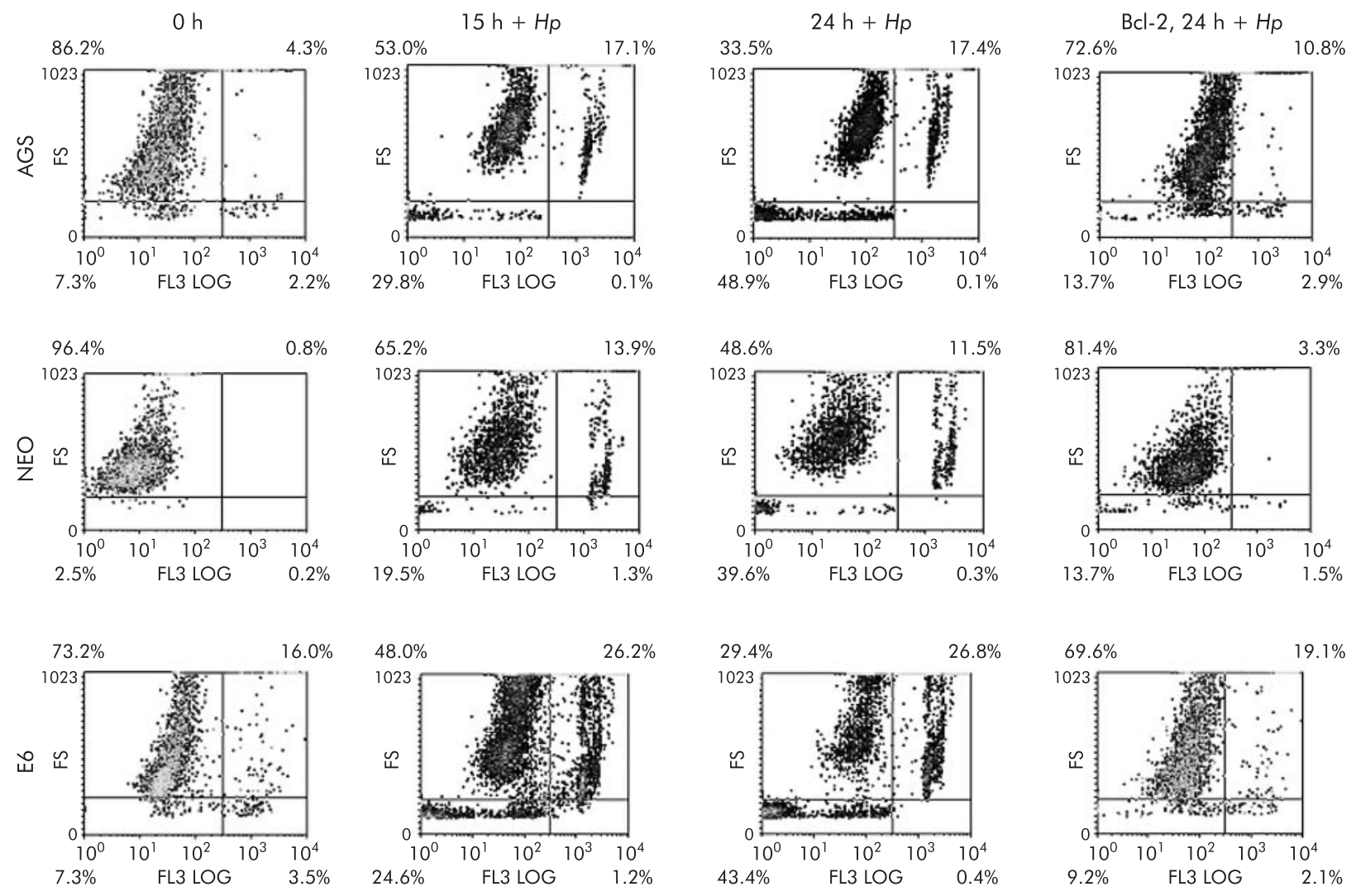

Figure 6 Cell viability decreased in gastric cells with forced Bcl-2 expression treated with Helicobacter pylori. AGS, AGS-E6 (E6), or AGS-neo (NEO) cells were cocultured with or without $H$ pylori for four hours. Data were accumulated by analysing an average population of 20000 cells by flow cytometry. In parallel experiments, AGS, AGS-E6, or AGS-neo cells transfected with Bcl-2 (to inhibit apoptosis) were stained as explained above. Viability was quantified by flow cytometry analysis of untransfected cells versus $\mathrm{Bcl}-2$ transfected gastric cells after $\mathrm{H}$ pylori treatment for the indicated time periods. Values represent the average of triplicate samples.

these cells rounded up to a lesser extent than their wild-type counterparts. Due to the limitations of confocal imaging of AGS cells, we also studied mitochondrial morphology during $H$ pylori induced apoptosis in another cell type. We found that wild-type MEF cells underwent dramatic mitochondrial fragmentation at seven hours after $H$ pylori exposure (fig 5). In these cells, a $43.9 \%(3.5 \%)$ increase in the percentage of cells with a fragmented mitochondrial phenotype was found (fig 5, images $\mathrm{A}, \mathrm{A}^{\prime}$ ) compared with a $10.4 \%$ (3.5\%) increase in ARF-null MEF cells (p53 function inactivated; fig 5, images $\left.\mathrm{B}, \mathrm{B}^{\prime}\right)$.

\section{$H$ pylori induces cell death, role of $\mathrm{Bcl}-2$, and caspase- 3 activation}

To further assess the death pathway targeting mitochondria, we measured viability using both PI and trypan blue exclusion dye. As shown in figs 6 and 7, viability began to decrease 2-3-fold after 7-24 hours of coculture of cells with $H$ pylori. Viability decreased in the p53 containing cell lines AGS and AGS-neo as well as in the p53 null AGS-E6 cells (fig 6). Hence $H$ pylori also induces p53 independent death effects which target the mitochondria. At time zero, $73 \%-86 \%$ of cells were viable. However, at 24 hours after $H$ pylori exposure, the number of viable cells decreased to $33.5 \%$ in AGS cells, $48.6 \%$ in AGS-neo cells, and 29.4\% in AGS-E6 cells. Treated cells were protected by overexpression of Bcl-2 in all three gastric cell lines and viability increased to $69-72 \%$ at 24 hours. Transfection efficiency of cells was very high with the Bcl-2, as determined by GFP expression. Therefore, Bcl-2 protects from $H$ pylori injury. To determine if blocking mitochondrial damage with Bcl-2 inhibits caspase-3 activity, we transiently expressed the antiapoptotic protein $\mathrm{Bcl}-2$ prior to incubation with $H$ pylori. Caspase-3 cleavage and activity is known to be involved in $H$ pylori induced apoptosis. ${ }^{36}$ As others have shown that $\mathrm{Bcl}-2$ protects mitochondria from Bax mediated damage, ${ }^{41}{ }^{42}$ we measured caspase- 3 activity in $H$ pylori exposed cells. Forced expression of Bcl-2 was able to inhibit caspase- 3 activity in cells exposed to $H$ pylori (fig 7). At 0 hours, $10-14 \%$ of cells were positive for caspase-3 activity. However, at four hours after $H$ pylori exposure, caspase activity increased to $60 \%$ in AGS cells, to $39 \%$ in AGS-neo cells, and to 35\% in AGS-E6 cells. Treated cells were protected by forced expression of Bcl-2 in all three gastric cell lines and caspase activity decreased to $14 \%$ at 24 hours (similar levels as untreated cells). Hence the death pathways induced by $H$ pylori in AGS cells involves activation of caspase- 3 and can be inhibited by the antiapoptotic protein Bcl-2.

\section{DISCUSSION}

In this study, we have shown that $H$ pylori infection promotes apoptosis mediated through Bax that can be inhibited by forced expression of Bcl-2. As a consequence of $H$ pylori infection, mitochondrial fragmentation results in subsequent Bax translocation, caspase activation, and cell death. Apoptosis is defined as an active physiological process of cellular self destruction with specific morphology and biochemical changes. ${ }^{44}$ Apoptosis and proliferation are tightly regulated processes within cells. Among apoptosis related genes, p53, Bax, and Fas are of particular importance. The involvement of the Fas pathway has been shown together with involvement of Bcl-2 proteins in $H$ pylori induced apoptosis. $^{745-48} \mathrm{P} 53$ and p14 ${ }^{\mathrm{ARF}}$ are well known for suppression of 

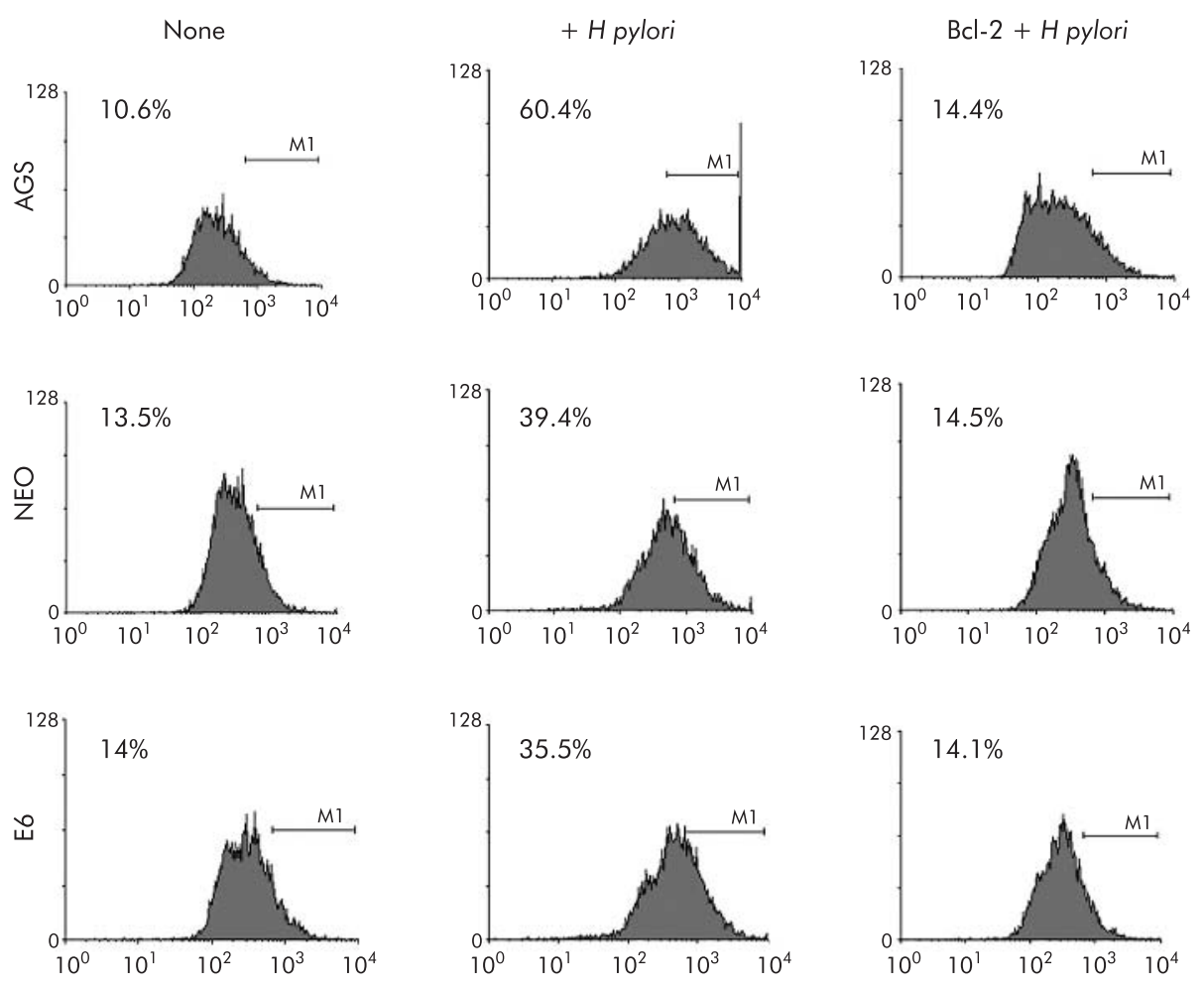

Figure 7 Caspase-3 activity was inhibited in gastric cells with forced Bcl-2 expression treated with Helicobacter pylori. AGS, AGS-E6 (E6), or AGSneo (NEO) cells were cocultured with or without $H$ pylori for four hours. Caspase-3 activity was measured with a peptide substrate for caspase-3 which when cleaved emits green fluorescence (excitation, $505 \mathrm{~nm}$; emission, $530 \mathrm{~nm}$ ). Data were accumulated by analysing an average population of 20000 cells by flow cytometry. In parallel experiments, AGS, AGS-E6, or AGS-neo cells transfected with Bcl-2 (to inhibit apoptosis) were stained as explained above. Caspase-3 activity was quantified by flow cytometry analysis of untransfected cells versus Bcl-2 transfected gastric cells after $\mathrm{H}$ pylori treatment for the indicated time periods. Bcl-2 transfected cells exhibited significantly reduced caspsase-3 activity four hours after $\mathrm{H}$ pylori treatment $(p<0.05)$. In contrast, untransfected cells exhibited much higher caspase-3 activity. Values represent the average of triplicate samples.

cellular proliferation. P53 induces G1 arrest, and can lead to apoptosis in response to DNA damaging agents in the absence of ARF. ${ }^{49-51}$ We previously demonstrated that $H$ pylori induces p $14{ }^{\mathrm{ARF}}$ in gastric epithelial cells. ${ }^{43} 14^{\mathrm{ARF}}$ stabilises the p53 protein in response to cellular stress which can result in cell cycle arrest, and also in apoptosis.

Cleavage of caspase-3, -8 , and cytochrome $c$ release occur in $H$ pylori induced apoptosis. ${ }^{36} 5253$ Cytochrome $c$ release from the mitochondria may contribute to activation of caspases. On entering the cytosol, cytochrome $c$ promotes the assembly of a multi-protein complex that induces proteolytic processing and activation of cell death by caspases. ${ }^{17}{ }^{54}$ Once activated by cytochrome $c$, caspase- 3 triggers activation of various important cellular substrates leading to apoptosis. ${ }^{19} 2955$

Bacteria other than $H$ pylori may have the ability to activate host apoptotic processes in response to infection and inflammation. ${ }^{56}$ Bax translocation has also been demonstrated in Chlamydia psittaci infected cells where it was found to be independent of caspase activation. ${ }^{57}$ It is not clear whether activation of the caspase pathway is generally beneficial to the host or pathogen. Some pathogens, including Chlamydia trachomattis and Rickettsia rickettsii, do not induce apoptosis, which may allow these organisms to grow and persist intercellularly. ${ }^{57}$ Streptococcus pyogenes causes mitochondrial dysfunction that leads to release of cytochrome $c$ from the mitochondria via Bax translocation. ${ }^{56}$

Release of cytochrome $c$ in response to $H$ pylori is an early event in gastric epithelial cells. $H$ pylori can cause stress signalling to transduce the mitochondria and cause the release of cytochrome $c^{5960}$ Activation of Bax and its translocation to the mitochondria followed by membrane depolarisation, mitochondrial fragmentation, and cytochrome $c$ release are associated with $H$ pylori induced apoptosis, which is consistent with various studies demonstrating decreased levels of Bcl-2 and increased levels of Bak in AGS cells exposed to $H$ pylori. ${ }^{2}$ When cytochrome $c$ is released, it binds together with dATP and apoptosis activating factor-1 to procaspase-9, resulting in the activation of downstream caspases. ${ }^{61}$

Previous publications confirmed cell death induction by $H$ pylori in the presence and absence of the Cag pathogenicity island. However, the presence of the both cagA and vacuolating cytotoxin may be important factors in causing the severe damage of the human gastric mucosa produced by $H$ pylori. ${ }^{62-64}$ While qualitative differences occur between cag positive and cag negative $H$ pylori strains, most studies show no significant difference in the ability of these strains to induce apoptosis or to stimulate proapoptotic factors. Therefore, our experiments used only a cag positive strain. In prior experiments we have shown that $H$ pylori induced apoptosis is associated with accumulation of $\mathrm{p} 53$ protein and a decrease in $\mathrm{Bcl}-2 .{ }^{8}$ In addition, we demonstrated ${ }^{36}$ that $H$ pylori induced apoptosis activated caspase- 8 and caspase- 3 which in turn degrade poly adenosine ribose polymerase (PARP) and DNA fragmentation factor 45, consistent with the data reported here.

Several lines of in vitro evidence suggest that induction of caspase-8, -9, and -3 may play a role in the aetiology of $H$ pylori associated ulcer disease and may be involved in early processes of gastric carcinoma. ${ }^{59}{ }^{65}$ After $H$ pylori infection, cytochrome $c$ release from the mitochondria activates caspase-8, -9 , and -3 , as confirmed by cleavage of PARP. ${ }^{59}{ }^{65}$ Different $H$ pylori cytotoxic factors including vacA, lipopolysaccharide, 
and/or the pathogenicity island may be important in Bax translocation and induction of cytochrome $c$ from mitochondria which induces oligomerisation of Apaf-1, allowing activation of procaspase-9 to promote apoptosis. ${ }^{61}$ The antiapoptotic BCL-2 family of proteins are generally characterised by the presence of four conserved $\mathrm{BH} 3$ domains (BHl-4). Bak-BH3 peptide induces Bax conformational change in vitro only in the presence of mitochondria and the outer mitochondrial membrane fraction is sufficient for induction of Bax conformational change. ${ }^{66} \mathrm{BAX}$ and BAK are designated as "multidomain" BCL-2 family proapoptotic proteins that share homology over three domains (BHl, $\mathrm{BH} 2$, and $\mathrm{BH} 3$ ) with BCL-2, and lack the fourth domain (BH4), which is unique to antiapoptotic proteins ${ }^{67}$. In contrast, several other proapoptotic proteins share only a single domain (BH3) with BCL-2 and are designated as "BH3-only" proteins. BH3-only proteins serve as effectors of apoptotic signalling and connect the various apoptotic stimuli with core apoptotic machinery. ${ }^{67}$ Both BAX and BAK appear to exist in inactive conformations and are "activated" in response to various apoptotic stimuli and form multimeric complexes. ${ }^{68}{ }^{69}$ Studies have led to the suggestion that both BAX and BAK constitute critical components of mammalian apoptosis machinery, and these multidomain proteins may be functionally overlapping. ${ }^{68}$ A recent study suggests that the multidomain proapoptotic proteins may not have fully overlapping functions in all cell types and that BAX may play a more important role in the apoptosis paradigms in human epithelial cancer cells. ${ }^{66}$ Therefore, Bax may play a critical role in $H$ pylori apoptosis and the role of other effectors such as Bim remains to be investigated. In addition, metabolic damage due to loss of mitochondrial function may contribute to $H$ pylori cell death.

Here, we demonstrated that forced expression of Bcl-2 not only blocks cell death but also inhibits caspase-3 activity. This indicates the significant mitochondrial involvement in $H$ pylori induced cell death. It would be an oversimplification however to assume that the pathway leading from mitochondrial membrane permeabilisation to nuclear apoptosis relies exclusively on caspase activation. The death pathway is initiated at the mitochondria by release of cytochrome $c$, a process that can be inhibited by antiapoptotic proteins of the Bcl-2 family. Thus translocation of Bax to the mitochondria in cells infected with $H$ pylori and subsequent mitochondrial fragmentation provide a direct link between infection and apoptosis induced by $H$ pylori.

In many cell types, p53 function is known to be critical for the ability to commit programmed cell death. Therefore, our data obtained from p53 deficient AGS-E6 and ARF-null MEF cells (both of which showed reduced apoptosis associated changes in their mitochondrial phenotypes) show that both p53 dependent (more evident in MEFs) and p53 independent pathways can lead to mitochondrial fragmentation in response to $H$ pylori induced cell damage. We conclude that during apoptosis induced by $H$ pylori infection, the mitochondrial morphology in respective cell types switches from a reticulotubular to a punctiform mitochondrial phenotype, compatible with organelle fragmentation.

In summary, $H$ pylori induces Bax to translocate to the mitochondria resulting in membrane depolarisation and mitochondrial fragmentation. These phenomena are reduced in cells lacking wild-type p53. The reduction in Bax translocation in cells lacking p53 varies among cell types. P53 cross talk with Bax in these cells may influence cell death through mitochondria fragmentation, but this is not critical as cells that lack wild-type p53 still undergo apoptosis, although less frequently. Therefore, p53 is partially involved in the death process in gastric cells exposed to $H$ pylori and alternative pathways are likely to play a role. Further studies are needed to identify bacterial factors responsible for stimulation of Bax translocation and/or p53 stabilisation that results in mitochondrial fragmentation and subsequent apoptosis.

\section{ACKNOWLEDGEMENTS}

This work was supported by PHS grant from the National Institutes of Health, DK53713 and DK56664.

\section{Authors' affiliations}

H Ashktorab, B Kifle, D T Smoot, Cancer Center and GI Division, Department of Medicine, Howard University, Washington, DC, USA

S Frank, National Institutes of Health, Bethesda, Maryland, USA

A R Khaled, S K Durum, National Cancer Institute-Frederick, Frederick, Maryland, USA

\section{REFERENCES}

1 Bechi P, Balzi M, Becciolini A, et al. Helicobacter pylori and cell proliferation of the gastric mucosa: possible implications for gastric carcinogenesis. Am J Gastroenterology 1996:91:271-6.

2 Chen G, Sordillo EM, Ramey WG, et al. Apoptosis in gastric epithelial cells is induced by Helicobacter pylori and accompanied by increased expression of BAK. Biochem Biophys Res Commun 1997;239:626-32.

3 Chow K, Bank S, Ahn J, et al. Helicobacter pylori infection does not increase gastric antrum mucosal cell proliferation. Am J Gastroenterology 1995;90:64-6.

4 Jones NL, Shannon PT, Cutz E, et al. Increase in proliferation and apoptosis of gastric epithelial cells early in the natural history of Helicobacter pylori infection. Am J Pathol 1997;151:1695-703.

5 Fan X, Crowe SE, Behar S, et al. The effect of class II major histocompatibility complex expression on adherence of Helicobacter pylori and induction of apoptosis in gastric epithelial cells: a mechanism for T helper cell type 1mediated damage. J Exp Med 1998;187:1659-69.

6 Moss S, Calam J, Agarwal B, et al. Helicobacter pylori infection induces gastric epithelial apoptosis in vivo. Gastro 1995;108:A171.

7 Konturek PC, Pierzchalski P, Konturek SJ, et al. Helicobacter pylori induces apoptosis in gastric mucosa through an upregulation of Bax expression in humans. Scand J Gastroenterol 1999;34:375-83.

8 Ahmed A, Smoot D, Littelton G, et al. Helicobacter pylori inhibits gastric cell cycle. Microbes Infect 2000;2:1-11.

9 Shirin H, Sordillo EM, Oh SH, et al. Helicobacter pylori inhibits the G1 to S transition in AGS gastric epithelial cells. Cancer Res 1999;59:2277-81.

10 Ashktorab H, Rees B, Allen C, et al. Regulation of apoptosis by differential expression of waf1, p53 and bcl2 in gastric epithelial cells exposed to Helicobacter pylori. Gastroenterology 1999:112:A59.

11 Le'Negrate G, Ricci V, Hofman V, et al. Epithelial intestinal cell apoptosis induced by Helicobacter pylori depends on expression of the cag pathogenicity island phenotype. Infect Immun 2001;69:5001-9.

12 Fan X, Gunasena H, Cheng Z, et al. Helicobacter pylori urease binds to class II MHC on gastric epithelial cells and induces their apoptosis. J Immunol 2000; 165: 1918-24.

13 Kawahara T, Teshima S, Kuwano Y, et al. Helicobacter pylori lipopolysaccharide induces apoptosis of cultured guinea pig gastric mucosal cells. Am J Physiol Gastrointest Liver Physiol 2001;281:G726-34.

14 Green DR, Amarante-Mendes GP. The point of no return: mitochondria, caspases, and the commitment to cell death. Results Probl Cell Differ 1998;24:45-61.

15 Green DR, Reed JC. Mitochondria and apoptosis. Science 1998;281:1309-12.

16 Patterson SD, Spahr CS, Daugas E, et al. Mass spectrometric identification of proteins released from mitochondria undergoing permeability transition. Cell Death Differ 2000;7:137-44.

17 Liu X, Kim CN, Yang J, et al. Induction of apoptotic program in cell-free extracts: requirement for dATP and cytochrome c. Cell 1996;86:147-57.

18 Cohen GM. Caspases: the executioners of apoptosis. Biochem J 1997:326:1-16

19 Salvesen GS, Dixit VM. Caspases: intracellular signaling by proteolysis. Cell 1997;91:443-6.

20 Nagahara Y, Ikekita M, Shinomiya T. Immunosuppressant FTY720 induces apoptosis by direct induction of permeability transition and release of cytochrome c from mitochondria. J Immunol 2000; 165:3250-9.

21 Yang J, Liu X, Bhalla K, et al. Prevention of apoptosis by Bcl-2: release of cytochrome c from mitochondria blocked. Science 1997;275:1129-32.

22 Kluck RM, Bossy-Wetzel E, Green DR, et al. The release of cytochrome c from mitochondria: a primary site for $\mathrm{Bcl}-2$ regulation of apoptosis. Science 1997;275:1132-6.

23 Korsmeyer SJ, Wei MC, Saito M, et al. Pro-apoptotic cascade activates BID, which oligomerizes $\mathrm{BAK}$ or $\mathrm{BAX}$ into pores that result in the release of cytochrome c. Cell Death Differ 2000;7:1166-73.

24 Vieira HL, Kroemer G. Pathophysiology of mitochondrial cell death control. Cell Mol Life Sci 1999;56:971-6.

25 Basanez G, Nechushtan A, Drozhinin O, et al. Bax, but not Bcl-xL, decreases the lifetime of planar phospholipid bilayer membranes at subnanomolar concentrations. Proc Natl Acad Sci U S A 1999:96:5492-7. 
26 Wolter KG, Hsu YT, Smith CL, et al. Movement of Bax from the cytosol to mitochondria during apoptosis. J Cell Biol 1997; 139:1281-92.

27 Basu A, Haldar S. The relationship between $\mathrm{Bcl} 2$, Bax and p53: consequence for cell cycle progression and cell death. Mol Hum Reprod 1998;4:1099-109.

28 Johnson MD, Wu X, Aithmitti N, et al. Peg3/Pwl is a mediator between p53 and Bax in DNA damage-induced neuronal death. J Biol Chem 2002;9:9.

29 Smaili SS, Hsu YT, Sanders KM, et al. Bax translocation to mitochondria subsequent to a rapid loss of mitochondrial membrane potential. Cell Death Differ 2001;8:909-20.

30 Hsu YT, Wolter KG, Youle RJ. Cytosol-to-membrane redistribution of Bax and $\mathrm{Bcl}-\mathrm{X}(\mathrm{L})$ during apoptosis. Proc Natl Acad Sci U S A 1997;94:3668-72.

31 Momand J, Zambetti GP. Mdm-2: "big brother" of p53. J Cell Biochem 1997:64:343-52

32 Pomerantz J, Schreiber-Agus N, Liegeois NJ, et al. The Ink4a tumor suppressor gene product, p19Arf, interacts with MDM2 and neutralizes MDM2's inhibition of p53. Cell 1998;92:713-23.

33 Kamijo T, Weber JD, Zambetti G, et al. Functional and physical interactions of the ARF fumor suppressor with $p 53$ and Mdm2. Proc Natl Acad Sci U S A 1998:95:8292-7.

34 Ashktorab H, Ahmed A, Littleton G, et al. p53 and p1 4 increase sensitivity of gastric cells to H pylori-induced apoptosis. Dig Dis Sci 2003;48:1284-91.

35 Hasumi K, Tanaka K, Saitoh S, et al. Roles of tumor necrosis factor-alphareceptor type 1 and Fas in the Helicobacter pylori-induced apoptosis of gastric epithelial cells. J Gastroenterol Hepatol 2002;17:651-8.

36 Ashktorab $\mathrm{H}$, Neapolitano $\mathrm{M}$, Bomma C, et al. In vivo and in vitro activation of caspase- 8 and -3 associated with Helicobacter pylori infection. Microbes Infect 2002:4:713-22.

37 Peek RM jr, Blaser MJ, Mays DJ, et al. Helicobacter pylori strain-specific genotypes and modulation of the gastric epithelial cell cycle. Cancer Res 1999;59:6124-31

38 Huibregtse JM, Beaudenon SL. Mechanism of HPV E6 proteins in cellular transformation. Semin Cancer Biol 1996:7:317-26.

39 Crook T, Vousden KH. Interaction of HPV E6 with p53 and associated proteins. Biochem Soc Trans 1994;22:52-5.

40 Eischen CM, Roussel MF, Korsmeyer SJ, et al. Bax loss impairs Myc-induced apoptosis and circumvents the selection of p53 mutations during Mycmediated lymphomagenesis. Mol Cell Biol 2001;21:7653-62.

41 Cheng EH, Wei MC, Weiler S, et al. BCL-2, BCL-X(L) sequester BH3 domainonly molecules preventing BAX- and BAK-mediated mitochondrial apoptosis. $\mathrm{Mol}$ Cell $2001 ; 8: 705-11$.

42 Zhang H, Holzgreve W, De Geyter C. Bcl2-L-10, a novel anti-apoptotic member of the Bcl-2 family, blocks apoptosis in the mitochondria death pathway but not in the death receptor pathway. Hum Mol Genet 2001;10:2329-39.

43 Ahmed A, Hassan A, Cornell A, et al. Alterations in MDM2 expression in gastric epithelial cells after exposure to Helicobacter pylori. Gastereoentrology 2001;114:A182.

44 Steller H. Mechanisms and genes of cellular suicide. Science 1995;267: 1445-9

45 Rudi J, Kuck D, Strand S, et al. Involvement of the CD95 (APO-1/Fas) receptor and ligand system in Helicobacter pylori-induced gastric epithelial apoptosis. J Clin Invest 1998;102:1506-14.

46 Houghton J, Korah RM, Condon MR, et al. Apoptosis in Helicobacter pyloriassociated gastric and duodenal ulcer disease is mediated via the Fas antigen pathway. Dig Dis Sci 1999:44:465-78.

47 Houghton J, Macera-Bloch LS, Harrison L, et al. Tumor necrosis factor alpha and interleukin 1 beta up-regulate gastric mucosal Fas antigen expression in Helicobacter pylori infection. Infect Immun 2000;68:1 189-95.

48 Jones NL, Day AS, Jennings HA, et al. Helicobacter pylori induces gastric epithelial cell apoptosis in association with increased Fas receptor expression. Infect Immun 1999;67:4237-42.
49 Linke SP, Harris MP, Neugebauer SE, et al. p53-mediated accumulation of hypophosphorylated $\mathrm{pRb}$ after the $\mathrm{Gl}$ restriction point fails to halt cell cycle progression. Oncogene 1997; 15:337-45.

50 Midgley CA, Owens B, Briscoe CV, et al. Coupling between gamma irradiation, p53 induction and the apoptotic response depends upon cell type in vivo. J Cell Sci 1995;108:1843-8.

51 Sherr CJ. The INK4a/ARF network in tumour suppression. Nat Rev Mol Cell Biol $2001 ; 2: 731-7$

52 Galmiche A, Rassow J, Doye A, et al. The N-terminal 34 kDa fragment of Helicobacter pylori vacuolating cytotoxin targets mitochondria and induces cytochrome c release. Embo J 2000;19:6361-70.

53 Maeda S, Yoshida H, Mitsuno Y, et al. Analysis of apoptotic and antiapoptotic signalling pathways induced by Helicobacter pylori. Gut 2002;50:771-8.

54 Zou H, Henzel WJ, Liu X, et al. Apaf-1, a human protein homologous to C. elegans CED-4, participates in cytochrome c-dependent activation of caspase3. Cell 1997;90:405-13.

55 Desagher S, Martinou JC. Mitochondria as the central control point of apoptosis. Trends Cell Biol 2000;10:369-77.

56 Nakagawa I, Nakata M, Kawabata S, et al. Cytochrome c-mediated caspase9 activation triggers apoptosis in Streptococcus pyogenes-infected epithelial cells. Cell Microbiol 2001;3:395-405.

57 Fan T, Lu H, Hu H, et al. Inhibition of apoptosis in chlamydia-infected cells: blockade of mitochondrial cytochrome $\mathrm{c}$ release and caspase activation. J Exp Med 1998; 187:487-96.

58 Clifton DR, Goss RA, Sahni SK, et al. NF-kappa B-dependent inhibition of apoptosis is essential for host cellsurvival during Rickettsia rickettsii infection. Proc Natl Acad Sci U S A 1998;95:4646-51.

59 Obst B, Wagner S, Sewing KF, et al. Helicobacter pylori causes DNA damage in gastric epithelial cells. Carcinogenesis 2000;21:1111-15.

60 Smoot DT, Elliott TB, Verspaget HW, et al. Influence of Helicobacter pylori on reactive oxygen-induced gastric epithelial cell injury. Carcinogenesis 2000;21:2091-5.

61 Ruiz-Vela A, Gonzalez de Buitrago G, Martinez AC. Nuclear Apaf-1 and cytochrome $c$ redistribution following stress-induced apoptosis. FEBS Lett 2002:517:133-8.

62 Kodama K, Fujioka T, lto A, et al. Toxigenicity of Helicobacter pylori isolates possessing cagA gene and vacuolating cytotoxin. J Gastroenterol 1998;33(suppl 10):14-17.

$63 \mathrm{Kim}$ JM, Kim JS, Jung HC, et al. Virulence factors of Helicobacter pylori in Korean isolates do not influence proinflammatory cytokine gene expression and apoptosis in human gastric epithelial cells, nor do these factors influence the clinical outcome. J Gastroenterol 2000;35:898-906.

64 Hua J, Zheng PY, Yeoh KG, et al. The status of the cagA gene does not predict Helicobacter pylori-associated peptic ulcer disease in Singapore. Microbios 2000;102: $113-20$

65 Shibayama K, Doi Y, Shibata N, et al. Apoptotic signaling pathway activated by Helicobacter pylori infection and increase of apoptosis-inducing activity under serum-starved conditions. Infect Immun 2001;69:3181-9.

66 Theodorakis $P$, Lomonosova $E$, Chinnadurai $G$. Critical requirement of BAX for manifestation of apoptosis induced by multiple stimuli in human epithelial cancer cells. Cancer Res 2002;62:3373-6.

67 Green DR. Apoptotic pathways: paper wraps stone blunts scissors. Cell 2000;102:1-4

68 Wei MC, Zong WX, Cheng EH, et al. Proapoptotic BAX and BAK: a requisite gateway to mitochondrial dysfunction and death. Science 2001;292:727-30.

69 Gross A, Jockel J, Wei MC, et al. Enforced dimerization of BAX results in its translocation, mitochondrial dysfunction and apoptosis. Embo $J$ $1998 ; 17: 3878-85$. 